\section{Zusammenhang von Asthma und Adipositas bei Kindern}

Contreras ZA et al. Does early-onset asthma increase childhood obesity risk? A pooled analysis of 16 European cohorts. Eur Respir J 2018; doi:10.1183/13993003.00504-2018

Asthma bronchiale und Adipositas gehören zu den wichtigsten chronischen Erkrankungen im Kindesalter und nehmen parallel zu. Übergewicht steigert das Risiko für ein Asthma bronchiale und Asthma-ähnliche Symptome. Aber wie ist es umgekehrt? Die Pool-Analyse aus 16 europäischen Kohorten spricht für eine bidirektionale Assoziation.

Für die Analyse lagen Daten von insgesamt 21130 Kindern mit Informationen zu einem möglichen Asthma bronchiale, den Phänotypen, anthropometrischen Daten und zahlreichen zusätzlichen Einflussvariablen vor. Bei der Ersterhebung waren die Patienten durchschnittlich 4,1 Jahre alt. Bis etwa zum 8 . Lebensjahr fanden im Mittel 3,2 Untersuchungen statt. Die Prävalenz eines ärztlich diagnostizierten Asthmas betrug 6,0\%. Wheezing lag bei $12,5 \%$ und ein frühes Wheezing bei $18,1 \%$ vor. $13,7 \%$ der Kinder hatten eine allergische Rhinitis. 483 Kinder entwickelten Übergewicht (2,3\%; Cole-International Obesity Task Force and sex specific cutoffs/WHO-Cutoffs). Die adjustierte, multivariate Analyse bestätigte eine höhere Wahrscheinlichkeit für eine Adipositas, wenn die Kinder von Asthma, Wheezing und/oder einer allergischen Rhinitis betroffen waren. Besondere Risikosteigerungen bestanden bei:

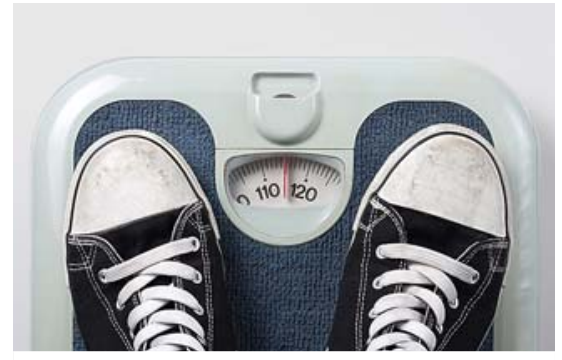

- Nach Angaben der Autoren erhöhen Atemprobleme im Kindesalter das Risiko später Übergewicht zu entwickeln. Bildquelle: tournee/stock.adobe.com

- Asthma bronchiale HR 1,66 (95\%-KI 1,18-2,33),

- aktives Asthma bronchiale HR 1,98 (95\%-KI 1,31-3,00),

- persistierendes Wheezing HR 1,51 (95\%-KI 1,08-2,09),

- Asthma ohne allergische Rhinitis HR 2,04 (95\%-KI 1,32-3,13).

Die Risikozunahme für Übergewicht betrug für Wheezing $29 \%$, für Wheezing im Kleinkindalter 22\%, für Late-onsetWheezing $12 \%$, vorübergehendes Wheezing $6 \%$ und die allergische Rhinitis $29 \%$.

Atemnot oder die Notwendigkeit der Medikamenteneinnahme waren ebenfalls mit einer inzidenten Adipositas assoziiert. Inhalative Kortikosteroide steigerten das Risiko um $42 \%$. Unter Berücksichtigung des Asthma-Status schwächte sich der Zusammenhang leicht ab und unterschritt das Signifikanzniveau. Die Adjustierung mit der Medikation hingegen änderte die positive Assoziation von Asthma bronchiale und Übergewicht nicht.
Die Adjustierung mit dem Ausgangs-BMI bestätigte die positive Beziehung von Asthma bronchiale und Adipositas (nicht signifikant). Das Geschlecht, Geburtsgewicht, elterliches Asthma, mütterliche Bildung, Asthmamedikation und Stillen beeinflussten die Interaktion nicht wesentlich.

FAZIT

Ein frühes Asthma bronchiale und Wheezing waren mit einem gesteigerten Adipositasrisiko assoziiert. Mit der allergischen Rhinitis bestand ein schwächerer Zusammenhang. Der zeitliche Verlauf bei der Entstehung der Erkrankungen bleibe offen. Die Autoren diskutieren u.a. genetische Gemeinsamkeiten, einen veränderten Signalweg von Chitinase-3-ähnlichem-Protein-1, eine Adipokindysregulation, die geringere körperliche Aktivität Erkrankter, Störungen des Mikrobioms und präpartale Expositionen.

\section{Dr. med. Susanne Krome, Melle}

